\title{
INVESTIGACIÓN
}

Recibido: 01/07/2021 --- Aceptado: 16/09/2021 --- Publicado: 03/01/2022

\section{UN ESTUDIO EXPLORATORIO SOBRE EL IMPACTO DEL NEUROMARKETING EN ENTORNOS VIRTUALES DE APRENDIZAJE}

\section{An exploratory study on the impact of neuromarketing on virtual learning environments}

Luis Manuel Cerdá Suárez: Universidad Internacional de La Rioja. España. luis.cerda@unir.net

Carmen Cristófol Rodriguez: Universidad Internacional de La Rioja. España. carmen.cristofol@unir.net

\section{Cómo citar el artículo:}

Cerdá Suárez, L. M. y Cristófol Rodriguez, C. (2022). Un estudio exploratorio sobre el impacto del neuromarketing en entornos virtuales de aprendizaje. Vivat Academia. Revista de Comunicación, 155, 1-16. http://doi.org/10.15178/va.2022.155.e1391

\section{RESUMEN}

El neuromarketing es un tópico fundamental en el mundo tecnológico actual y ha experimentado un crecimiento explosivo en los últimos años como herramienta de la comunicación. Últimamente, las asignaturas de neuromarketing han mejorado mucho cuando la enseñanza está respaldada por cursos y experimentos de laboratorio siguiendo el paradigma de "aprender haciendo", que proporciona a los estudiantes una comprensión más profunda de su aprendizaje. Sin embargo, muchos programas educativos no enseñan a los estudiantes sobre el uso y las aplicaciones del neuromarketing. Bajo el supuesto de que los avances en neuromarketing cambiarán las prácticas tradicionales en el aula, el objetivo de este trabajo es proponer una combinación de tecnologías para convertir un proyecto de neuromarketing en una actividad de laboratorio, haciendo que este sea más atractivo para los estudiantes al mejorar la aplicación de los planes de estudio en postgrados de administración de empresas. Este proyecto ha sido evaluado con éxito sobre la base de respuestas a cuestionarios de estudiantes y expertos que calificaron positivamente la actividad de laboratorio, encontrando el aprendizaje como muy bueno y/o excelente, alcanzándose además buenos resultados académicas. En el contexto específico de una universidad privada virtual, este trabajo se orientó al diseño de un taller de neuromarketing para desarrollar determinadas competencias genéricas en la mejora de los procesos educativos en las universidades. Los hallazgos de esta investigación 
resultan relevantes en las decisiones de política educativa, pero también en la teoría y práctica pedagógica en el ámbito de este estudio.

PALABRAS CLAVE: Gestión del conocimiento - Neuromarketing - Innovación docente - Procesos de negocio - Educación Superior - Enseñanza virtual - Aprender haciendo.

\section{ABSTRACT}

Neuromarketing is an essential topic in the current technological world, and it has experienced an explosive growth in the last years as a tool for communication. Nowadays, neuromarketing subjects have greatly improved when classroom teaching is supported by adequate laboratory courses and experiments, following the 'learning by doing' paradigm, which provides students with a deeper understanding of theoretical lessons. However, many postgraduate programmes do not teach their students about the use and applications of neuromarketing. It is believed that developments in neuromarketing will likely change the traditional practices in the classroom. The objective of this paper is to propose a mix of consumer-based technologies to develop a neuromarketing project into a laboratory activity. These technologies can make neuromarketing more appealing to students by enhancing the attractiveness of business administration curricula. This neuromarketing exploration project has been evaluated successfully based on the results and responses to questionnaires: students and experts rated the neuromarketing laboratory activity highly. Students found the laboratory activity learning in the neuromarketing exploration project to be very good or excellent. Moreover, the students obtained good academic outcomes. Within the specific context of a virtual private university, this work was oriented to design a neuromarketing workshop in order to develop certain generic competencies for improving educational processes at universities. The findings of this research will be relevant in decisions of educational policy, but also on the pedagogical theory and practice in the scope of this study.

KEYWORDS: Knowledge management - Neuromarketing - teaching innovation business process - Higher Education - virtual learning - learning-by-doing.

\section{UM ESTUDO EXPLORATÓRIO SOBRE O IMPACTO DO NEUROMARKETING EM ENTORNOS VIRTUAIS DE APRENDIZADO}

\section{RESUMO}

O neuromarketing é um tópico fundamental no mundo tecnológico atual e tem acontecido um crescimento explosivo nos últimos anos como ferramenta de comunicação. Ultimamente as matérias de neuromarketing tem melhorado muito quando o processo de ensino está respaldado por cursos e experiências laboratoriais 
seguindo o paradigma de aprender fazendo, que proporciona aos estudantes uma compreensão mais aprofundada do seu próprio aprendizado. Porém, muitos programas educativos não ensinam aos estudantes sobre o uso e as aplicações do neuromarketing. Sob a suposição de que os avanços no neuromarketing mudaram as práticas tradicionais na aula, o objetivo deste trabalho é propor uma combinação de tecnologias para transformar um projeto de neuromarketing em uma prática de laboratório, fazendo que este seja mais atrativo para os estudantes ao melhorar a aplicação dos planos de estudo em pós graduações de administração de empresas.

Este projeto tem sido avaliado com sucesso sobre a base de respostas de questionários de estudantes e experts que qualificaram positivamente a atividade de laboratório, encontrando o aprendizado como muito bom e/o excelente, atingindo além disso bons resultados acadêmicos. No contexto específico de uma universidade privada virtual, este trabalho se orientou a criação de um workshop de neuromarketing para desenvolver determinadas competências genéricas orientadas a melhora dos processos de pesquisa. As descobertas desta pesquisa resultam relevantes nas decisões da política educativa, mas também na teoria e prática pedagógica no âmbito deste estudo.

\section{PALAVRAS CHAVE}

Gestão do conhecimento - Neuromarketing - Inovação pedagógica - Processos de negócio - Educação Superior - Ensino virtual - Aprender fazendo.

\section{INTRODUCCIÓN}

El neuromarketing no es una disciplina tradicional, sino que puede entenderse como una combinación de disciplinas que involucran conocimientos y herramientas de marketing, administración de empresas, psicología, sociología, ingeniería y tecnología, entre otras disciplinas relevantes. Es más, el neuromarketing ofrece aplicaciones que desarrollan la forma en que se supervisa al individuo, la manera en que se evalúa el entorno y el enfoque en que se mejora la experiencia del cliente (Cerdá, 2016). Por estas razones y como enfoque, esta disciplina ha atraído enorme atención en los últimos años en muchos procesos de negocio y productos derivados de áreas de ingeniería y negocios, a través del diseño y dispositivos ópticos y magnéticos, pantallas y tecnologías médicas; herramientas todas ellas que facilitan su utilización en experimentos de laboratorio (Casado-Aranda et al., 2019; Kaklauskas et al., 2020).

El neuromarketing como disciplina ofrece una forma relevante de aprender a ver las oportunidades que surgen cuando convergen varios campos de las ciencias sociales, la tecnología y la ingeniería (Asrar-ul-Haq et al., 2017; Cerdá, 2016). Cada año se publican cientos de artículos en este campo con desarrollos de nuevas tecnologías; tanto en versiones y formatos virtuales, como impresos. En este sentido, cabe apreciar que el intercambio de conceptos, herramientas y su aplicación y enseñanza en cursos de diversas disciplinas y áreas de relevancia, puede facilitar una mayor accesibilidad a la tecnología y la ciencia en particular (Avinash et al., 2018).

Vivat Academia. Revista de Comunicación. 2022, n 155, 1-16 
Sin embargo, todavía no abundan ni se han desarrollado suficientes cursos y seminarios de aprendizaje basados en la aplicación del neuromarketing. Con frecuencia, la enseñanza del neuromarketing a estudiantes universitarios de ciencias sociales puede hacer uso de laboratorios educativos prácticos, pero los destinatarios de estos cursos argumentan en muchas ocasiones que los experimentos de neuromarketing son delicados, de disponibilidad limitada y costosos de mantener (Salehzadeh, et al., 2020). Por tanto, el conocimiento sobre las mejores prácticas en neuromarketing está poco extendido; de manera que todavía la educación superior actual se enfrenta a cuestiones fundamentales a evaluar en este ámbito (Chihiro Watanabe et al., 2019; Papanastasiou et al., 2020).

Diversos estudios académicos en el ámbito han destacado la relevancia de hacer uso de experiencias prácticas para integrar el neuromarketing en el currículo académico. Por esta razón, los experimentos y las experiencias previas con la actividad de laboratorio facilitan la introducción de módulos prácticos, como un impulso importante de la educación empresarial y en ciencias sociales (Chen, 2016; Seligman et al., 2009). Profundizando en lo anterior, el desarrollo práctico de un curso de formación superior en neuromarketing, utilizando instrumentos y dispositivos experimentales en un laboratorio, ha sido valorado positivamente en la literatura. En concreto, esto se ha estudiado para cursos con un número significativo de tareas y actividades, en las que los estudiantes puedan verificar y practicar todos los conceptos y métodos teóricos mediante el desarrollo de cursos y seminarios (Blömeke y Olsen, 2019).

Es decir, los laboratorios de módulos y cursos de neuromarketing configuran un entorno académico importante para los estudiantes, y facilitan su formación en profundidad particularmente en el ámbito de la educación superior. Esto es importante en el aprendizaje práctico de un laboratorio: así, se ha reportado con frecuencia que la participación cognitiva en el aprendizaje es un indicador validado de logros y comportamiento proactivo en educación; en especial, en disciplinas propias del ámbito de la ingeniería y los negocios (Dirican, 2015; Golnar-Nik et al., 2019).

En el contexto específico de una universidad privada virtual, este trabajo está orientado a desarrollar determinadas competencias genéricas para mejorar los procesos educativos en las universidades. El esquema de este documento es el siguiente: el primer apartado hace referencia a la introducción y las motivaciones relacionadas con esta investigación. En segundo lugar, se revisa la literatura sobre estrategias y enfoques didácticos del aprendizaje experiencial en la Educación Superior, orientados a promover proyectos de neuromarketing en los procesos de enseñanza y aprendizaje. En tercer lugar, en este artículo se describe un método sistemático para implementar estas prácticas en el aula: en este contexto, se presenta una metodología sistemática para recopilar y analizar datos. En cuarto lugar, se lleva a cabo una discusión de los resultados. Finalmente, el último apartado está dedicado

Vivat Academia. Revista de Comunicación. 2022, n 155, 1-16 
a las conclusiones, implicaciones en el aula y futuras investigaciones. Los hallazgos de esta investigación serán relevantes en las decisiones de política educativa, pero también en la teoría y práctica pedagógica en el ámbito de este estudio.

\section{OBJETIVOS}

La actividad de laboratorio es una de las experiencias más relevantes para los estudiantes de pregrado, ya que les permite observar y explorar aplicaciones de teorías fundamentales y conectar teoría y práctica, desarrollando una comprensión sólida y profunda de lecciones teóricas. Varias investigaciones han señalado la relevancia de utilizar la experiencia práctica para integrar el neuromarketing en el laboratorio en los planes de estudio de pregrado.

Por ejemplo, en un estudio relevante sobre este tópico de estudio, los estudiantes emplearon su conocimiento colectivo para utilizar dispositivos en neuromarketing; todo ello, con la finalidad de desarrollar nuevos experimentos modulares. También se utilizaron métodos pedagógicos para introducir a los estudiantes en el campo de la investigación en el laboratorio (Zhang, et al., 2020). Además, la instrucción de laboratorio, particularmente en una disciplina como el neuromarketing, puede ayudar a los estudiantes a desarrollar habilidades experimentales y la capacidad de trabajar en equipo (Tshewang et al., 2016). Este tipo de instrucciones didácticas también facilita la introducción de planes de estudio sistemáticos, que pueden ayudar de manera eficaz a los estudiantes a alcanzar una comprensión holística y significativa; todo ello, con la aplicación de un mapa de conocimientos y la enseñanza integrada del neuromarketing y las prácticas de laboratorio en el plan de estudios (Folwarczny et al., 2019).

Diversos autores han integrado dispositivos físicos prácticos mediante un grupo de módulos de aprendizaje en neuromarketing interrelacionados entre sí. Profundizando en esto, autores como Wang y Hsu (2014) describieron en su trabajo un curso de pregrado de descubrimiento de prácticas de neuromarketing enriquecido con algunos módulos de esta disciplina y experimentos prácticos (Siddiqui et al., 2019). Todos estos proyectos destacan especialmente porque están a favor de introducir módulos prácticos de neuromarketing experimental en el contexto de las aulas, como forma práctica de mejorar el aprendizaje.

La mayor parte de la literatura citada en este estudio ha confirmado que incluir cursos de laboratorio en la enseñanza puede conducir a una mejor comprensión y una conceptualización más clara de conceptos e instrumentos para cursos en diversas disciplinas y áreas de relevancia; y, al mismo tiempo, ofrece una mayor accesibilidad al aprendizaje del neuromarketing como disciplina académica. Como se mencionó anteriormente, durante las actividades de laboratorio en el aprendizaje de esta disciplina -y con la finalidad de involucrar a los estudiantes con experiencias de aprendizaje mejoradas-, resulta oportuno prestar más atención a los procesos, en lugar del mero resultado del uso de la tecnología en las aulas (Luiz et al., 2020).

Vivat Academia. Revista de Comunicación. 2022, n 155, 1-16 
Este estudio enfatiza el apoyo para la enseñanza que pueden proporcionar las actividades de neuromarketing, y presenta un proyecto de estudio de caso que se realiza en la actividad de laboratorio en cursos de neuromarketing (Moghadam y Seyyedsalehi, 2018). El objetivo principal del curso de proyectos de exploración de neuromarketing ha consistido en contribuir al desarrollo de habilidades de comprensión del neuromarketing en la creación y prueba de actividades efectivas en los estudiantes, en el contexto del aprendizaje en sus propias aulas. En este sentido, en línea con estudios recientes, el propósito del estudio que aquí se cita ha sido el de explorar las actividades de planificación, diseño, desarrollo, análisis y evaluación que los estudiantes encontraron al implementar sus proyectos en un ambiente de laboratorio (Granziera y Perera, 2019).

En este estudio, la actividad experimental en un laboratorio es un recurso importante para los estudiantes, porque proporciona métodos alternativos para resolver problemas o dilemas mediante el diseño, la crítica y la evaluación de situaciones específicas (Jang, 2019). Es decir, este método incorpora la concepción de "aprender haciendo", ya que los estudiantes son responsables de planificar e implementar sus ideas y soluciones; al mismo tiempo, invita a los alumnos a buscar información, diseñar y probar diferentes soluciones (Gutiérrez, 2019).

Según revela la literatura académica sobre estos aspectos, actividades como estas podrían ayudar a los estudiantes a construir mejor el conocimiento, especialmente en campos en los que el comportamiento y la respuesta de los elementos reales en el experimento resultan muy importantes (Mañas-Viniegra et al., 2020). En este estudio y como se verá a continuación, el curso de laboratorio en el módulo experimental cubre el procesamiento, junto con la evaluación a partir del examen de las propiedades mecánicas de los instrumentos utilizados.

\section{METODOLOGÍA}

Además de los módulos en clase y sobre contenidos prácticos, se pidió a los estudiantes participantes que prepararan un trabajo final sobre una aplicación específica de los módulos de neuromarketing (Karakus et al., 2015). Los trabajos se enviaron individualmente cubriendo temas como propiedades ópticas, mecánicas, etc. Además, se utilizó la evaluación de expertos, la autoevaluación y la evaluación del profesor; todo ello, para que los estudiantes y los profesores pudieran hacer modificaciones durante el proyecto para tener el mayor éxito académico posible. Los informes intermedios fueron evaluados para ayudar a los profesores a verificar el trabajo. La evaluación final se basó en la documentación completa y, los hallazgos del proyecto, en la actividad de laboratorio del curso (de acuerdo con el detalle que se indica en la tabla 1).

En este estudio, la actividad de laboratorio creó una situación de aprendizaje a partir de la configuración de un enfoque integral de la enseñanza y el 
aprendizaje en el aula. El instructor diseñó el curso basado en proyectos, utilizando una actividad de laboratorio para que los alumnos pudieran construir sus propios conocimientos y habilidades; todo ello, experimentando varias situaciones de aprendizaje. Dentro de este marco, los estudiantes podían analizar estudios previos, crear un plan, realizar investigaciones relacionadas y compartir nuevos conocimientos.

El curso estuvo basado en las actividades de neuromarketing que aquí se describen, dentro del plan de estudios de la Universidad Internacional de La Rioja; y proporcionó a los alumnos las habilidades requeridas en el plan de estudios, mediante el desarrollo de un aprendizaje basado en proyectos. Para completar esta actividad académica, los estudiantes debieron realizar lo siguiente:

1) definir los problemas y tareas de la asignatura;

2) investigar una situación e implementar el proyecto propuesto por el profesor en el aula;

3) proporcionar comentarios y revisar el proyecto;

4) presentar el proyecto en forma escrita y oral; $y$

5) describir los hallazgos y evaluar el trabajo.

En este experimento, la actividad se llevó a cabo durante una programación de 14 semanas como parte de un curso de seis créditos, en el que se inscribieron los estudiantes universitarios. Para analizar los datos, se aplicó esta investigación en el contexto de una universidad virtual española, implementando estilos de aprendizaje en los que los estudiantes fueron interrogados por los profesores sobre sus opiniones tras el desarrollo y los resultados del curso, mediante la recopilación y el análisis posterior de sus percepciones sobre las actividades desarrolladas. En esta investigación se consideraron explícitamente dos aspectos del proceso metodológico, tal y como se describen a continuación: 1) las fuentes; y 2) el procedimiento detallado de la investigación.

En primer lugar y respecto de las fuentes, se implementó una metodología de triangulación en dos líneas de aplicación: 1) revisión bibliográfica de los temas presentados; y 2) una encuesta de opinión de los estudiantes, recogida en un cuestionario estructurado de campos disciplinares como Neuromarketing y Administración de Empresas (o Negocios, en general).

De acuerdo con las condiciones de esta investigación exploratoria, los informantes fueron seleccionados de una universidad virtual debido a que estas experiencias pueden revelar algunas percepciones significativas para obtener hallazgos relevantes sobre estos temas; en particular, la mayor dificultad inherente en experimentos de laboratorio en este ámbito. Se aplicó una escala diseñada previamente a partir de la literatura académica, con la finalidad evidente de medir estilos de enseñanza a estudiantes aplicada; en este caso específico, en el contexto de la Universidad Internacional de La Rioja (UNIR, 
España). Los participantes rellenaron un cuestionario en línea relacionado con sus experiencias y situaciones particulares de aprendizaje como manera de aproximación al contexto de aprendizaje; previamente, se aplicaron varias entrevistas en profundidad sobre el target (aunque por razones expositivas, en este trabajo solo se evidencian los resultados cuantitativos), y profesores con experiencia en Educación Superior analizaron la validez de la herramienta aquí descrita.

En segundo lugar, los estudiantes fueron seleccionados de un censo. Se implementó un diseño igualmente aleatorio, en términos del planteamiento de un estilo de enseñanza transaccional versus transformacional a implementar por los profesores, con respecto al experimento de laboratorio. Los estudiantes completaron después del curso el cuestionario, que comprendía de dos secciones diferentes para recopilar la información: Parte I: "Desempeño del profesor en el aula"; y Parte II: "Datos de identificación y clasificación". En la Parte I del cuestionario, la escala de medición requería valoraciones en escala Likert desde 1 hasta 7; mientras que la Parte II del cuestionario se midió con variables nominales, ordinales y de razón (esta última, para una variable como la edad, medida en años).

Tabla 1. Metodología de la investigación

\begin{tabular}{|c|c|c|c|}
\hline FASE & ANÁLISIS & METODOLOGÍA & TÉCNICA \\
\hline & $\begin{array}{l}\text { Bibliografía } \\
\text { Validez aparente }\end{array}$ & Revisión de literatura & Análisis documental \\
\hline Validación & $\begin{array}{l}\text { Fiabilidad } \\
\text { Validez de } \\
\text { constructo }\end{array}$ & $\begin{array}{l}\text { Investigación cualitativa } \\
\text { y cuantitativa (análisis de } \\
\text { fiabilidad global y } \\
\text { validez factorial) }\end{array}$ & $\begin{array}{l}\text { Análisis descriptivo de las } \\
\text { variables, y análisis de } \\
\text { contenido } \\
\text { Alpha de Cronbach, } \\
\text { correlación item-total } \\
\text { Exploratory/confirmatory } \\
\text { factor análisis factorial } \\
\text { exploratorio y confirmatorio, } \\
\text { test T }\end{array}$ \\
\hline
\end{tabular}

Fuente: Elaboración propia

El trabajo de campo en la Universidad Internacional de La Rioja se realizó en mayo de 2020. La Tabla 1 muestra los detalles de cada paso del experimento, de acuerdo con el cronograma diseñado. Los constructos e instrumentos del curso de neuromarketing se explicaron primero a los alumnos a través de una conferencia, y luego se les dio instrucciones para el desarrollo del experimento a través de la actividad de laboratorio. La actividad del experimento se compuso de dos grandes procedimientos: 1) planificación de las tareas de aprendizaje; y 2) evaluación de los resultados del experimento. Al final del experimento, los estudiantes recibieron retroalimentación de varios informantes y se 
administraron otras medidas como la escala de desempeño y la evaluación de la actividad de neuromarketing.

\section{RESULTADOS Y DISCUSIÓN}

En términos comparativos, la Tabla 2 muestra el perfil de los estudiantes que participaron en este estudio en función de los dos principales estilos de enseñanza analizados, es decir, estilo de enseñanza transaccional versus transformacional, con respecto al experimento de neuromarketing implementado.

Tabla 2. Muestra total en la Universidad Internacional de La Rioja

\begin{tabular}{|l|c|c|c|}
\hline \multicolumn{1}{|c|}{ MUESTRA } & $\begin{array}{c}\text { Estilo } \\
\text { transaccional }\end{array}$ & $\begin{array}{c}\text { Estilo } \\
\text { transformacional }\end{array}$ & TOTALES \\
\hline Edad del entrevistado: & 25.2 & 31.4 & 28.2 \\
- Media & 3.42 & 4.32 & 3.83 \\
\hline Género del entrevistado: & & & \\
- Femenino & $55 \%$ & $45.2 \%$ & $50 \%$ \\
- Masculino & $45 \%$ & $54.8 \%$ & $50 \%$ \\
\hline ESTUDIANTES TOTALES & $\mathbf{3 0}$ & $\mathbf{3 0}$ & $\mathbf{6 0}$ \\
\hline
\end{tabular}

Fuente: Elaboración propia

En este punto, conviene resaltar la presencia de diferencias significativas entre estudiantes y si esta consideración tiene o no relevancia en el estudio; todo ello, con la finalidad de concluir sobre las implicaciones de esta investigación. Por ejemplo, en términos descriptivos, solamente resulta apreciable que, en el grupo donde el profesor aplicó un estilo transaccional en el aula, hay más estudiantes mujeres que hombres, pero se observa lo contrario cuando se implementó el estilo transformacional del profesor. El número total de participantes fue de 60.

\section{Actividad de neuromarketing}

En este estudio, se pudieron configurar módulos experimentales en neuromarketing mediante el diseño de contenidos que incluyeron una interfaz para experimentos e instrucciones. Los estudiantes utilizaron dos categorías de contenidos para preparar sus programas para sus módulos, y la primera consideración en el análisis fue con el fin de diseñar dimensiones para evaluar el estado del arte sobre el desempeño del profesor en el aula.

De acuerdo con la metodología descrita en la última sección, el análisis documental identificó insights y categorías sobre este constructo, según diversos trabajos de investigación que han sido analizados para este estudio. Así, se revisó la literatura de acuerdo con el siguiente procedimiento: en primer lugar, se analizó el 
marco conceptual sobre el desempeño del profesor en el aula y, en segundo lugar, se enfocó el análisis en el detalle de la evaluación del contenido de este experimento.

Para promover un estilo transformacional de enseñanza en el aula -siguiendo lo indicado por diversos autores, como Nussbaum et al. (2012)-, los participantes entrevistados para este trabajo señalaron que, tanto la inteligencia emocional como la satisfacción, resultan dos indicadores importantes para comprender el desempeño del profesor en el aula.

\section{Validación de la escala y análisis comparativo}

Con el fin de comprender los resultados descritos en este trabajo, se presenta en este apartado la identificación del constructo respecto del desempeño académico, en términos de un enfoque cuantitativo que fue la base de obtención de información y su análisis para esta investigación. Además, se observó la consistencia interna particular de la escala de desempeño (Alfa de Cronbach) y la confiabilidad general de este instrumento de medición, que mostró un valor Alfa de 0.883 (todos los ítems tenían una distribución aproximadamente normal).

Los resultados de este análisis estadístico mostraron evidencias empíricas significativas. Además, un análisis detallado de la Tabla 3 evidenció que la correlación ítem-total osciló entre 0,851 y 0,917. Para validar las características psicométricas de esta escala, se aplicó un análisis factorial exploratorio de componentes principales con rotación Varimax, y se realizó un análisis factorial confirmatorio para redefinir aún más la escala de medición. Para este análisis confirmatorio, se observó la adecuación de la estructura factorial a través de CFI, RMSEA y SRMR.

La escala del desempeño del profesor en el aula se obtuvo en términos de la puntuación media de la suma de los ítems que miden este constructo. Para evidenciar la importancia del estilo particular de enseñanza relacionado con el desempeño del profesor en el aula, se realizó después un análisis t-test comparando por tipo de enseñanza (es decir, estilo transaccional versus transformacional). En cuanto a la puntuación media de la escala, se encontró una diferencia significativa en el desempeño del profesor (ver Tabla 3: valor $\mathrm{p}<0.05$ ).

Tabla 3. Impacto del tipo of enseñanza sobre el desempeño del profesor en el aula

\begin{tabular}{lccccc}
\hline \multicolumn{5}{c}{ Medias por tipo de estilo de enseñanza } \\
\cline { 3 - 6 } VARIABLE & $\begin{array}{c}\text { Media } \\
\text { (Desv. Tip.) }\end{array}$ & $\begin{array}{c}\text { Estilo } \\
\text { transformacional }\end{array}$ & $\begin{array}{c}\text { Estilo } \\
\text { transaccional }\end{array}$ & $\boldsymbol{t}$ & $\boldsymbol{p}<\mathbf{0 . 0 5}$ \\
\hline $\begin{array}{l}\text { Escala de } \\
\text { desempeño }\end{array}$ & $4.95(0.87)$ & 5.975 & 3.834 & 4.362 & 0.001 \\
\hline
\end{tabular}




\section{Fuente: Elaboración propia}

En cuanto al coeficiente Alfa de Cronbach de la actividad del laboratorio de neuromarketing, los resultados del cuestionario mostraron que los niveles de satisfacción de los expertos y estudiantes en este estudio fueron 0,851 y 0,917, respectivamente. Estos resultados demostraron la oportuna fiabilidad de la coherencia interna de la encuesta. Además, más del 75\% de los estudiantes -aquellos con puntuaciones superiores a 80- encontraron muy bueno o excelente el aprendizaje de la actividad de laboratorio en el proyecto de neuromarketing; alcanzándose también buenas calificaciones y elevados grados de satisfacción.

Según el instrumento descrito por autores diversos (Nussbaum et al., 2012), en este constructo las cargas factoriales expresaron que los valores superaron el 0,850 para ítems específicamente relacionados con "métodos didácticos que encuentro satisfactorios", "el profesor nos premia por los logros. . . "," El profesor es eficaz para satisfacer las necesidades. . . "Y" nuevas formas de motivar al grupo". También se probaron la confiabilidad y validez de la escala de desempeño: la prueba Alfa de Cronbach, la inter-correlación de los ítems y el análisis factorial exploratorio determinaron la estructura de un factor de esta escala. Estos hallazgos determinaron que era posible reducir el número total de ítems de esta escala y presentar una escala de un factor sobre el desempeño del profesor en el aula (Cerdá, 2016).

A partir de lo anterior, los resultados revelan que la escala de desempeño se vio afectada por el tipo de enseñanza (es decir, el estilo transformacional versus transaccional del profesor). Abundando en la importancia de los resultados de este trabajo, los hallazgos evidenciados en esta investigación muestran que la escala está relacionada con el estilo particular de enseñanza. La revisión preliminar de la literatura estudiada en el contexto de esta investigación confirma estos resultados y sugiere que los profesores que brindan atención a sus estudiantes en un estilo transformacional pueden lograr niveles similares de participación y satisfacción de los estudiantes, con cierta independencia del contexto que se tenga en cuenta (Cerdá, 2016; Nussbaum et al., 2012).

Para concluir la evidencia de los resultados obtenidos en este caso de estudio, esta investigación permitió además apreciar el efecto positivo de las acciones de enseñanza transformacional sobre el desempeño del docente en el aula, en términos de satisfacción. Un total de sesenta estudiantes completaron finalmente el cuestionario con ítems relacionados con experiencias y acciones relevantes en el aula virtual y el análisis de los resultados sugiere que los estudiantes son capaces de distinguir actividades e intereses según sus motivaciones personales, y el estilo particular de enseñanza aplicado por el profesor en el aula. Además, la principal contribución de este estudio revela que las experiencias impactan positivamente en el desempeño en el aula y, en definitiva, permite evidenciar que medir las experiencias y acciones positivas se vuelve fundamental para mejorar el aprendizaje; en particular, en el contexto de experiencias de aprendizaje en neuromarketing.

Vivat Academia. Revista de Comunicación. 2022, n 155, 1-16 


\section{CONCLUSIONES}

En la actividad de laboratorio, como revela la literatura y se ha podido apreciar en este estudio, los cursos de neuromarketing que utilizan una plataforma de laboratorio y los proyectos de exploración en este ámbito potencian el interés de los estudiantes, y ofrecen conocimientos susceptibles de aplicación práctica. El estudio abordó la reciente implementación de un experimento en un curso de neuromarketing e incluso, el proyecto también incursionó en los planes de estudio actuales mediante la incorporación de laboratorios, la orientación de los estudiantes en la investigación y el diseño de un conjunto de actividades de laboratorio para apreciar cómo el experimento mejoró diversas competencias y habilidades en el aula.

Ciertamente, el neuromarketing como disciplina, ha atraído la atención como proceso de desarrollo de productos en materias de ingeniería, administración de empresas y desarrollo de negocios: dispositivos ópticos y magnéticos, pantallas y tecnologías médicas. Recientemente, se aprecia en general una orientación hacia enfoques más tecnológicos en las instituciones educativas basados en el conocimiento colaborativo, la realidad virtual y los dispositivos digitales entre los estudiantes. En este sentido, la implementación de actividades de laboratorio en las instituciones educativas es una cuestión interesante, en particular orientada a una acción eficaz para el cambio en la educación. Es más: la puesta en práctica de experimentos ha generado ventajas y oportunidades de aprendizaje de los estudiantes en diversas instituciones; sin embargo, resulta evidente que los modelos aplicados tienden a ser reportados durante procesos comerciales y referidos a actividades de reingeniería; es decir, rara vez apoyan la implementación de los procesos. En la actividad de laboratorio, una plataforma de tecnologías para el desarrollo de proyectos de neuromarketing puede ayudar a los estudiantes a comprender los conceptos y la aplicación de sistemas basados en el enfoque hacia el consumidor.

Este trabajo tuvo como objetivo describir la aplicación de experimentos relacionados con neuromarketing en la Universidad Internacional de La Rioja, (UNIR, España). En particular, en esta investigación se han identificado algunas variables que juegan un papel activo en esta institución educativa, sin embargo, estos hallazgos en particular se pueden generalizar también a otros contextos educativos.

Las principales aportaciones relacionadas con los fundamentos conceptuales y gerenciales derivadas de este estudio empírico están orientadas a mejorar diversas actividades experimentales, cuando profesores e instructores implementan diversas tareas en el aula. Algunos indicadores -como la satisfacción de los estudiantes y las recomendaciones sobre oportunidades de mejora- son de especial relevancia para facilitar buenas prácticas en el aula -como se desprende de los resultados de este trabajo. 
Respecto a los objetivos específicos descritos en este documento, sus hallazgos refuerzan la importancia de revisar continuamente ciertas habilidades manejando diferentes aspectos de aprendizaje en el laboratorio. Como principal contribución de este trabajo, destaca la oportunidad de comprender esta investigación como un marco de referencia con aplicación a contextos similares, considerando ciertos aspectos específicos -entorno presencial, virtual...- de cada contexto en particular. En este sentido, este repositorio de conocimiento que se desprende del estudio empírico incluye diferentes experiencias reveladas por los estudiantes; todo ello, entendido como hallazgos y mejores prácticas en el aula universitaria. Además, estos resultados contribuyen a reforzar la propuesta de valor de las universidades en diferentes aspectos de los procesos centrados en el aprendizaje, así como de los planes de estudio, relacionados con la disciplina de neuromarketing en particular.

Adicionalmente, algunos aspectos significativos a considerar en términos de limitaciones de este estudio aquí abordado son los siguientes: en esta investigación, con alumnos de distintos países, se aprecia la dificultad para gestionar actores derivados de diversas culturas y visiones de todos los alumnos involucrados en la Universidad Internacional de La Rioja, de acuerdo con su misión y presencia geográfica en el mundo.

Gestionar la calidad del servicio en la docencia representa, además de todo lo expuesto anteriormente, un desafío importante en la gestión de las organizaciones e instituciones educativas. El repositorio de conocimiento generado por esta investigación facilitará la generalización de estos resultados y hallazgos, sirviendo como referencia para la extrapolación de estos resultados a futuras investigaciones.

\section{REFERENCIAS}

Asrar-ul-Haq, M., Anwar, S. y Hassan, M. (2017). Impact of emotional intelligence on teacher's performance in higher education institutions of Pakistan. Future Business Journal, 3(2), 87-97; http://dx.doi.org/10.1016/j.fbj.2017.05.003

Avinash, T., Dikshant, L. y Seema, S. (2018). Methods of Neuromarketing and Implication of the Frontal Theta Asymmetry induced due to musical stimulus as choice modeling. Procedia Computer Science, 132, 55-67, https://doi.org/10.1016/j.procs.2018.05.059

Blömeke, S. y Olsen, R. V. (2019). Consistency of results regarding teacher effects across subjects, school levels, outcomes and countries. Teaching and Teacher Education, 77, 170-182. https://doi.org/10.1016/j.tate.2018.09.018

Casado-Aranda, L-A., Dimoka, A. y Sánchez-Fernández, J. (2019). Consumer Processing of Online Trust Signals: A Neuroimaging Study. Journal of Interactive Marketing, 47, 159-180. https://doi.org/10.1016/j.intmar.2019.02.006

Vivat Academia. Revista de Comunicación. 2022, nº 155, 1-16 
Cerdá Suárez, L. M. y Cristófol Rodriguez, C.

Un estudio exploratorio sobre el impacto del neuromarketing en entornos virtuales de aprendizaje

Cerdá, L. M. (2016). Happiness in teaching: positive emotions for evaluating the relationship between leadership style and performance of the professor in the classroom. En Proceedings of 10th annual International Technology, Education and Development Conference INTED 2016. Valencia, March, 1396-1405. http://doi.org: $\underline{10.21125 / \text { inted.2016.1305 }}$

Chen, J. (2016). Understanding teacher emotions: The development of a teacher emotion inventory. Teaching and Teacher Education, 55, 68-77. http://doi.org: 10.1016/j.tate.2016.01.001

Chihiro Watanabe, C., Naveed, K. y Neittaanmäki, P. (2017). Co-evolution between trust in teachers and higher education toward digitally-rich learning environments. Technology in Society, 48, 70-96. https://doi.org: $\underline{10.1016 / j . t e c h s o c .2016 .11 .001}$

Dirican, C. (2015). The Impacts of Robotics, Artificial Intelligence on Business and Economics. Procedia - Social and Behavioral Sciences, 195, 564-573; https://doi.org/10.1016/j.sbspro.2015.06.134

Folwarczny, M., Pawar, S., Sigurdsson, V. y Fagerstrøm, A. (2019). Using neuroIS/consumer neuroscience tools to study healthy food choices: a review. Procedia Computer Science, 164, 532-537. https://doi.org/10.1016/j.procs.2019.12.216

Golnar-Nik, P., Farashi, S. y Safari, M.-S. (2019). The application of EEG power for the prediction and interpretation of consumer decision-making: A neuromarketing $\begin{array}{llllll}\text { study. Physiology } & \mathcal{E} & \text { Behavior } & \text { 207, }\end{array}$ https://doi.org/10.1016/j.physbeh.2019.04.025

Granziera, H. y Perera. H. N. (2019). Relations among teachers' self-efficacy beliefs, engagement, and work satisfaction: A social cognitive view. Contemporary $\begin{array}{lll}\text { Educational Psychology, 58, } & \text { 75-84. }\end{array}$ http://dx.doi.org/10.1016/j.cedpsych.2019.02.003

Gutiérrez, G. (2019). Neuromarketing as an effective tool for education in sales and advertising. Revista Latina de Comunicación Social, 74, 1173-1189. https:// dx.doi.org/10.4185/RLCS-2019-1377

Jang, H.-R. (2019). Teachers' intrinsic vs. extrinsic instructional goals predict their classroom motivating styles. Learning and Instruction, 60, 286-300; https://doi.org/10.1016/j.learninstruc.2017.11.001

Kaklauskas, A., Abraham, A., Dzemyda, G., Raslanas, S., Seniut, M., Ubarte, I., Kurasova, O., Binkyte-Veliene, A. y Cerkauskas, J. (2020). Emotional, affective and 
Cerdá Suárez, L. M. y Cristófol Rodriguez, C.

Un estudio exploratorio sobre el impacto del neuromarketing en entornos virtuales de aprendizaje

biometrical states analytics of a built environment. Engineering Applications of Artificial Intelligence. 91, 103621. https:// doi.org/10.1016/j.engappai.2020.103621

Karakus, O., Howard-Jones, P. A. y Jay, T. (2015). Primary and Secondary School Teachers' Knowledge and Misconceptions about the Brain in Turkey. Procedia Social and Behavioral Sciences, 174, 1933-1940. https://doi.org/10.1016/j.sbspro.2015.01.858

Luiz, I., Annukka Kim Lindell, A. K. y Ekuni, R. (2020). Neurophilia is stronger for educators than students in Brazil. Trends in Neuroscience and Education, 20, 100136. https://doi.org/10.1016/j.tine.2020.100136

Mañas-Viniegra, L., Núñez-Gómez, P. y Tur-Viñes, V. (2020). Neuromarketing as a strategic tool for predicting how Instagramers have an influence on the personal identity of adolescents and young people in Spain. Heliyon, 6, (3), e03578. https:// doi: 10.1016/j.heliyon.2020.e03578

Moghadam, S. M. y Seyyedsalehi, S. A. (2018). Nonlinear analysis and synthesis of video images using deep dynamic bottleneck neural networks for face recognition. Neural Networks, 105, 304-315. https:// doi.org/10.1016/j.neunet.2018.05.016

Nussbaum, P. A., Herrera, A., Joshi, R. y Hargraves, R. (2012). Analysis of Viewer EEG Data to Determine Categorization of Short Video Clip. Procedia Computer Science, 158-163. http:/ / doi.org: 10.1016/j.procs.2012.09.047

Papanastasiou, G., Drigas, A., Skianis, C. y Lytras, M. (2020). Brain computer interface based applications for training and rehabilitation of students with neurodevelopmental disorders. A literature review. Heliyon, 6(9), e04250; https://doi.org/10.1016/j.heliyon.2020.e04250

Salehzadeh, A., Calitz, A. P. y Greyling, J. (2020). Human activity recognition using deep electroencephalography learning. Biomedical Signal Processing and Control, 62, 102094; https://doi.org/10.1016/j.bspc.2020.102094

Seligman, M., Ernstb, R., Gillhamc, J., Reivicha, K y Linkins, M. (2009). Positive education: positive psychology and classroom interventions. Oxford Review of Education, 35(3), 293-311. https://doi.org/10.1080/03054980902934563

Siddiqui, N., Gorard, S. y See, B. H. (2019). Can learning beyond the classroom impact on social responsibility and academic attainment? An evaluation of the Children's University youth social action programme. Studies in Educational Evaluation, 61, 74-82. http://doi.org: 10.1016/j.stueduc.2019.03.004

Tshewang, R., Chandra, V. y Yeh, A. (2016). Students' and teachers' perceptions of classroom learning environment in Bhutanese eighth-grade mathematics classes.

Vivat Academia. Revista de Comunicación. 2022, nº 155, 1-16 
Cerdá Suárez, L. M. y Cristófol Rodriguez, C.

Un estudio exploratorio sobre el impacto del neuromarketing en entornos virtuales de aprendizaje

Learning Environments Research, 1(1), 1-20. https://doi.org/10.1007/s10984-016$\underline{9225-6}$

Wang, C.-C. y Hsu, M.-C. (2014). An exploratory study using inexpensive electroencephalography (EEG) to understand flow experience in computer-based

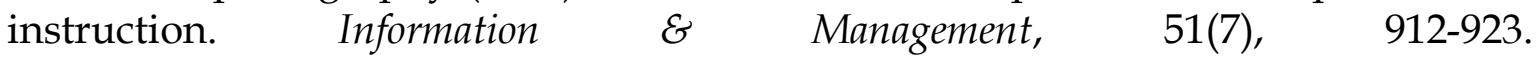
https://doi.org/10.1016/j.im.2014.05.010

Zhang, J., Yin, Z., Chen, P. y Nichele, S. (2020). Emotion recognition using multimodal data and machine learning techniques: A tutorial and review. Information Fusion, 59, 103-126. https:// doi.org: 10.1016/j.inffus.2020.01.011

\section{AUTORES:}

\section{Luis Manuel Cerdá Suárez}

Universidad Internacional de La Rioja. España. Sus ámbitos de trabajo se extienden en disciplinas propias de marketing, investigación de mercados, procesos empresariales, liderazgo y administración de empresas, tecnologías de la información y sistemas de gestión empresarial, en general. Además, ha sido profesor visitante en convenios postdoctorales con la Agencia Española de Cooperación Internacional para el Desarrollo (AECID), y ha impartido cursos y conferencias en España, México, Colombia, Ecuador, Estados Unidos, Chile y Portugal. También ha publicado capítulos de libro, libros, artículos en revistas y ponencias en congresos nacionales e internacionales, colaborando activamente en diversos comités científicos. Así mismo, dispone de diversos premios nacionales e internacionales de reconocimiento a su labor de investigación, acreditada con Sexenio de Investigación por la Comisión Nacional Evaluadora de la Actividad Investigadora, de la Agencia Nacional de Evaluación de la Calidad y Acreditación (ANECA).

ORCID ID: https://orcid.org/0000-0002-3909-8805

\section{Carmen Cristófol Rodriguez}

Universidad Internacional de La Rioja. España. Doctora en Comunicación y Licenciada en Publicidad y Relaciones Públicas (UMA). Participa como IP en Proyectos de Innovación Docente, como evaluadora en revistas de reconocido prestigio, y como miembro de tribunales de tesis y trabajos fin de máster. Editora de sección de Revista Mediterránea de Comunicación, investigadora en los grupos COMPUBES (Comunicación y Públicos Específicos) y IICCXXI (Industrias Culturales Hoy), ambos de la Universidad de Alicante. Es socia de la AEIC (sección 10) y AIRRPP. Forma parte del Programa de Doctorado Interuniversitario. Tiene experiencia docente en universidades públicas y privadas y en docencia virtual y presencial. Durante 17 años ha compaginado su labor docente e investigadora con su faceta como profesional de los medios de comunicación. Cuenta con un sexenio de investigación.

ORCID ID: https://orcid.org/0000-0001-9766-6500 
Cerdá Suárez, L. M. y Cristófol Rodriguez, C.

Un estudio exploratorio sobre el impacto del neuromarketing en entornos virtuales de aprendizaje 